\title{
IMAGE SEARCH ENGINE USING SIFT ALGORITHM
}

\author{
Joshi Parag ${ }^{1 *}$, Shinde Saiprasad Prakash², Panse Mihir ${ }^{3}$, Vaidya Omkar ${ }^{4}$, \\ ${ }^{* 1}$ Assistance Professor and ${ }^{2,3,4}$ Students Department \\ *1,2,3,4 Computer Engineering, Rajendra Mane College of Engineering and Technology, Ambav, Devrukh, Ratnagiri- \\ 415612. \\ 1,2,3, *Mumbai University \\ *1parag_joshi2007@yahoo.co.in, ${ }^{2}$ saishinde08@gmail.com, ${ }^{3}$ mihirpanse92@gmail.com \\ ${ }^{4}$ omkar.vaidya.4@gmail.com
}

\section{*Corresponding Author: -}

Email ID-parag_joshi2007@yahoo.co.in

\begin{abstract}
: -
The approach of SIFT feature detection taken in our implementation is similar with the one taken by Lowe, which is used for object recognition. According to Lowe's work, the invariant features extracted from images can be used to perform reliable matching between different views of an object or scene. The features can be different from image rotation and scale and robust across a substantial range of various distortion, addition of various other colors, and change in actual view of the image. The approach is efficient on feature extraction and has the ability to identify large numbers offeatures .In short changes image has will not be mined by our process in order to match the image where basically images are going to be matched using Histogram and RGB values of the image present in the Database of Admin i.e Search Engine itself and the image asked by the User to searched
\end{abstract}

Keywords: - Histogram, RGB values, Comparison of two images based on Histogram, Image Database, Threshold, Experimental Results

\section{(사 (요 (1)}




\section{INTRODUCTION}

Image recognition is becoming an increasingly important for many applications including human- machine interfaces, multimedia, security, communication. One of the most difficult problems is that the process of distinguishing a person from his image appearance has to be performed differently for particular image, because there are so many conflicting factors altering appearance of that image. Our implementation focuses on basically all features from an image and trying using these features to perform the image search. The approach of our feature detection taken in our implementation is similar with the one taken by Lowe which is used for recognition of object. According to our work, the invariant features extracted from images can be used to perform matching or comparison between different views of an object or scene of that image.

\section{Proposed Method}

Number of Images will already be present in the Database with their Descriptions, Basically the whole system developed will search the image User wants to search .But developing Copy of one of those images would be modified or edited to make it blur, change its Contrast, Brightness add some noises etc. Now that Image we upload on the search engine we designed. Modified image will get transferred to the image database original image, If the Modified image able to find its original Copy, it will view the image with the description about that Image system to check for the original image, If the Modified image able to find its original Copy, it will display the image with the description about that Image respectively.

\subsection{Histogram:}

The best technique for image search in a collection of heterogeneous images is the comparison of images based on their histograms. The histogram basically describes the gray-level or color distribution for a desired given image. It is a universal feature that can actually be used to perform a fast but no actually reliable indexing process. The histogram feature can be used as an initial step for database indexing so that in order to reduce the number of images for the next step which could use other features to compare the database images with a given query for matching image. The main advantage histogram actually provides is small sensitivity to scale, rotation and translation. An appropriation of color space, a color quantization scheme, a histogram representation, and a similarity metric are the main elements required for the design of a histogram based

Search Engine

\subsection{RGB:}

In RGB color model, each colour appears basically in its primary spectral components that is Red, Green, and Blue. The colour of a pixel is actually made up of three components that are red, green, and blue (RGB), described by their corresponding intensities respectively. Colour components are also known as colour channels. In the RGB colour model, a colour image can be represented by the intensity function.

$\mathrm{I} \mathrm{RGB}=(\mathrm{FR}, \mathrm{FG}, \mathrm{FB})$

Where $\operatorname{FR}(x, y)$ is the intensity of the pixel $(x, y)$ in the red channel, $\operatorname{FG}(x, y)$ is the intensity of pixel $(x, y)$ in the green channel, and $\operatorname{FB}(\mathrm{x}, \mathrm{y})$ is the intensity of pixel $(\mathrm{x}, \mathrm{y})$ in the blue channel. The intensity thus of each colour channel is basically stored using 8 bits, which therefore indicates that the quantization level is 256 .

\subsection{Proposed Methodology:}

> Proposed system here actually selected 10000 image databases with same Histogram and RGB values.

> Proposed system basically extracts all images features separately R, G, B values and Histogram values for problem Solving.

$>$ Proposed system implements features like color histogram, color projections

$>$ Mathematical approaches basically are mean, median and standard deviation are proposed for Good searching implementation

$>$ Proposed work of ours actually provides platform to extract images from the database using user image given in file uploader.

\section{Working Implementation of Search Engine}

3.1 Working of Search Engine:

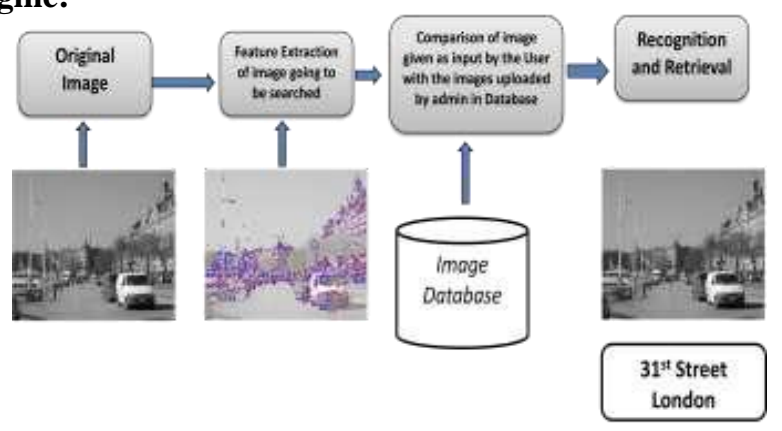

Figure 1: Flow Chart of Working of Image Search Engine 


\section{Image Uploaded In Database :}

The Implementation Of Search Engine works in different phases where actually the admin is also having the rights where the images uploaded by the Admin are directly stored in the Database and where the actual Database is Formed. The process to upload the file in the Database is given through the File Uploader Handler where the code to upload the file is being given. This was all about the Uploading Data of the Database from which the image can be fetched and the user can retrieve image when asked to search.

\section{Image Uploaded By User To Search :}

The image Uploaded by the User is the image user wants actually to Search in the Database. Here also the Function of File Uploader Handler is Used to Upload the image in the System so that the image can be searched

\section{Image Uploaded Stored in the TEMP Location:}

The image User uploaded using File Uploader Handler was actually stored in the TEMP loacation because no Database was being created by the Admin as the Temporary Loacation is granted in order to provide the search history later.

\section{Histogram And RGB values Of Image Uploaded to Search:}

The Image uploaded by the User which is first Stored in the TEMP location is set to find out the desired values of Histogram and RGB such that these values where first going to be used to Retrieve the image from the Database. The Histogram values and RGB values are totally Depended on the Height and Width of the image where the image is going to be matched pixel by pixel depending on the Height and Width of the image

\section{Histogram And RGB values Of Images Stored In The Database till the Matching Of The Image Uploaded by} the User:

The Image uploaded by the admin is Stored directly in the Database. Now when the image is present to find out the Histogram and RGB values the image is thus present to compare the Histogram and RGB values Founded out at that particular Interval. To program it, for loop of the List os Items is formed i.e. Number of Items present in the Database is the Number of times it will be compared; the only point is as soon as the image is matched the For Loop exits. The Relevant Image thus is found out to which it is compared Respectively

\section{Comparison Of Histogram and Giving out the Relevant Result :}

The images whose Histogram is matching to the Histogram of the image uploaded by the user is Compared in order to find out the accurate result. Though the Result obtained where quite relevant and also the Result obtained totally depend on the Threshold value as the Threshold value if given is $100 \%$ then only the accurate image will be found whereas the image whose Threshold value is less than $100 \%$ will not be matched if the Threshold is not allowed to Display.

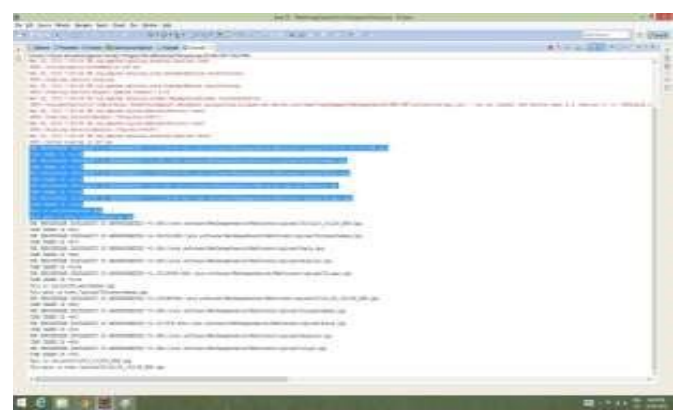

Figure 2: Accuracy Counted of every Images Giving the $100 \%$ accuracy as output

\section{Experimental Result}

The proposed method of histogram creation is being compared with the various traditional histogram creation approach. Their performances of finding similar images in a heterogeneous collection of images have been used as the comparison criterion where though the process of searching being is the longest the Accuracy is though the most Important of all. While Experimenting all the Results we Obtained were as follows: where the Image Rotated , Image Matched even when the scratches were present where the Robustness property being Resolved, Also the image with Resolution difference were obtained as the Result 


\subsection{Rotational Image Result:}

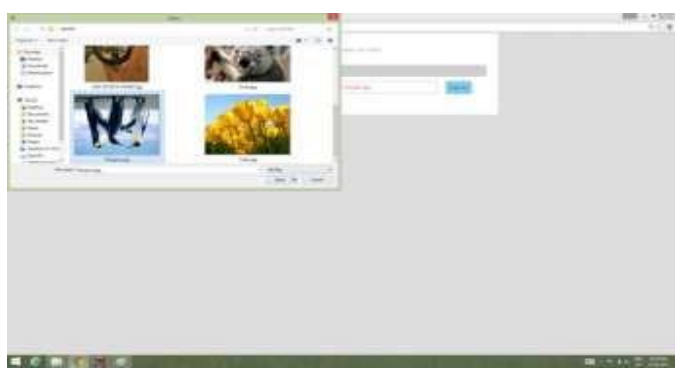

Figure 3: Rotated Image given as Input

\section{Result}

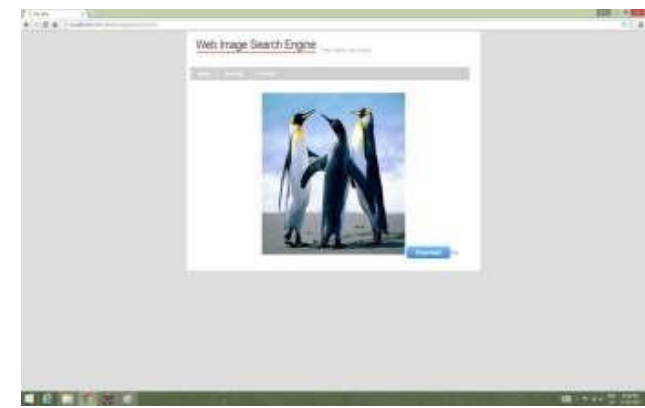

The Rotated Image is given as input as you can see (Figure3), here the image rotated is being given as input to the search engine through the user upload where the image to be searched is being given as input. Also in the next Image we can see that the Image i.e Original Image with its actual orientation is given as output as shown in next image(Figure 4).Here the property of rotational Image can be searched is proved where the search engine doesn't bother of image being Rotated

\section{Lower Image Resolution Result:}

The Lower Resolution Image is given as input as you can see (Figure5), here the image with lower resolution is being given as input to the search engine through the user upload where the image to be searched is being given as input. Also, in the next Image we can see that the Image i.e Original Image with its actual orientation and actual Resolution i.e about $5 \mathrm{mb}$ is given as output as shown in next image (Figure 6)

Here the property of Lower Resolution Image can be searched is proved where the search engine doesn't bother of image of whatever size it may be.

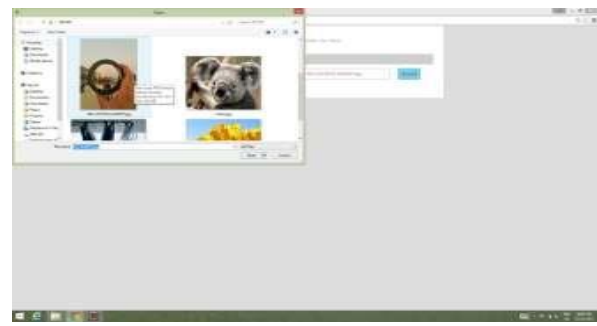

Figure 5: Image of lower Resolution Given as Input

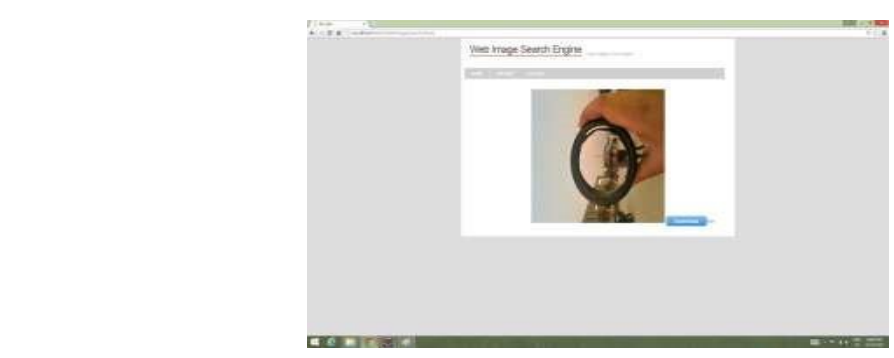

Figure 6: Original Image of Higher Resolution in Database Given as Output

\subsection{Scratched Image Result:}

The Scratched Image is given as input as you can see (Figure7), here the image Scratched where some elements are being added is been given as input to the search engine through the user upload where the image to be searched is being given as input. Also, in the next Image we can see that the Image i.e Original Image with its actual orientation and also without the extra elements is given as output as shown in next image (Figure 8) 
Here the property of Scratched Image can be searched is proved where the search engine doesn't bother of image being added by some extra elements whatever or however the image is being given as input, it will no doubt give the original image as the output

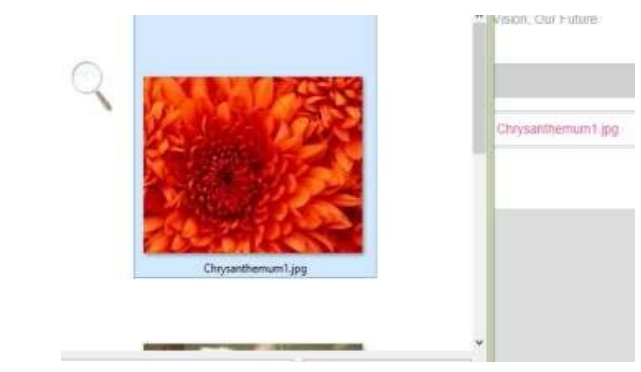

Figure 7: Image Scratched Given as Input

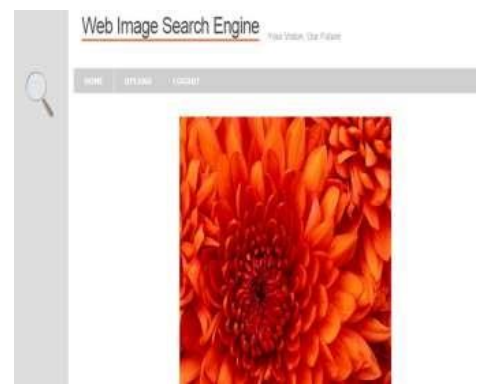

Figure 8: Original Image of same in Database Given as Output

\section{Conclusion}

Basically we found out in our Research that images are compared and match on some query of images being similar for example where the system automatically finds images similar to an input image. However, some different types of queries are also important. For example, it is also often important to search images in which particular object appears. So accordingly, we thought that our approach to this problem might be to generalize histogram accordingly of every image and also to separate pixels based on spatial coherence, or some other local property. Such that in mere sense where image matching is the most important tast done by this system. Here in this system, we basically have made the system in such a manner that though image is being Rotated or being Added by some Noise or any minor changes being done by user to check the accuracy, no doubt the user is going to find out the desirable Results for sure.

\section{ACKNOWLEDGEMENT}

We would like to express our sincere gratitude towards my guide, Prof. Joshi Parag, for the help, guidance and encouragement, he provided during the whole project. This work would have not been possible without his valuable time, patience and motivation. We thank him for making our stint thoroughly pleasant and enriching. It was great learning and an honor being his students. We are deeply indebted to the entire team in our Computer Department. They supported us with scientific guidance, advice and encouragement, they were always helpful and enthusiastic and this inspired us in our work.

\section{References}

[1].Zhuozeng Wang; Yalei Mei; Fang Yan (2009, 8 November), 'Web Image Search Engine Using SIFT Algorithm'. IEEE International conference on Web Information Systems and Mining, 2009. WISM 2009. Pp. 366-370, 2009.

[2].Nabeel Younus Khan, Brendan McCane, and Geoff Wyvill, "SIFT and SURF Performance Evaluation against

[3].Various Image Deformations on Benchmark Dataset”, International Conference on Digital Image Computing: Techniques and Applications, pp.501-506, 2011.

[4].ViniVidyadharan, and SubuSurendran, "Automatic Image Registration using SIFT-NCC", Special Issue of International Journal of Computer Applications (0975 - 8887) , pp.29-32, June 2012.

[5]. Luo Juan, and Oubong Gwun, "A Comparison of SIFT, PCA-SIFT and SURF", International Journal of Image Processing (IJIP), Vol. 3, Issue 4, pp. 143-152.

[6].Herbert Bay, Tinne Tuytelaars, and Luc Van Gool, “SURF: Speeded Up Robust Features”, pp. 1-14.

[7].Seok-Wun Ha, Yong-Ho Moon, "Multiple Object Tracking Using SIFT Features and Location Matching", International Journal of Smart Home Vol. 5, No. 4,pp. 1726, October 2011.

[8].D. Lowe. "Distinctive Image Features from ScaleInvariant Keypoints", Accepted for publication in the International Journal of Computer Vision, pp. 1-28, 2004.

[9].Hongbo Li, Ming Qi and Yu Wu, “A Real-Time Registration Method Of Augmented Reality Based On Surf And Optical Flow”, Journal Of Theoretical And Applied Information Technology, Vol. 42, No.2, pp. 281-286, August 2012 